\title{
El espacio urbano desde la educación y el currículo de arquitectura
}

\section{Urban space from an educational point of view and the architecture curriculum}

\author{
RODRIGUEZ MACHUCCA, José F. ${ }^{1}$
}

AYALA GARCIA, Erika T. ${ }^{2}$

CORONEL RUIZ, LUZ K. ${ }^{3}$

\begin{abstract}
Resumen
Se reconocen las categorías que sustentan el currículo del programa de Arquitectura de la Universidad Francisco de Paula Santander en materia de espacialidad urbana. Se utilizó una metodología mixta, exploratoria, con análisis documental y observación. Se encontró que el estudio de la temática del espacio urbano por medio de herramientas pedagógicas en el ciclo básico, de profundización y profesionalización favorece el análisis y la comprensión de categorías como emplazamiento, morfología, estética, usos, funciones urbanas, movilidad, temporalidad, técnica, tectónica y patrimonio.

Palabras clave: arquitectura, currículo, educación, espacio urbano.

Abstract

This research recognizes the categories that support the curriculum of the Architecture program of the Francisco de Paula Santander University in terms of urban spatiality. A mixed, exploratory methodology was used, with documentary analysis and observation. As a result, it was found that the study of the urban space theme through pedagogical tools in the basic cycle, deepening and professionalization favors the analysis and understanding of categories such as location, morphology, aesthetics, uses, urban functions, mobility, temporality, technique, tectonics and heritage.

key words: architecture, curriculum, education, urban space.
\end{abstract}

\section{Introducción}

Los programas de pregrado propios de las Instituciones de Educación Superior (IES) dentro del desarrollo de su quehacer académico, deben realizar constantemente procesos de autoevaluación, encaminados al mejoramiento continuo de la educación. Esto por medio de estrategias de mejora, trabajo participativo y discusión académica (Universidad Nacional de Colombia, 2014), a través de los cuales se puedan fortalecer el diseño curricular y los procesos de aprendizaje de los programas (Perilla Granados, 2018). Desde esta perspectiva, el programa de Arquitectura de la Universidad Francisco de Paula Santander (en adelante UFPS) ha

\footnotetext{
${ }^{1}$ Supervisor de Comunicaciones y Diseño de Samaritan's Purse Colombia. Grupo de Investigación Taller de Arquitectura y Gestión del Territorio TAR_GET Categoría B Colciencias Ferodriguez095@hotmail.com

2 Ph.D Teoria e Historia de la Arquitectura de la Universidad Politecnica de Cataluña- España, Directora Grupo de Investigación Taller de Arquitectura y Gestión del Territorio TAR_GET Categoria B Colciencias, ORCID http://orcid.org/0000-0001-7570-6639 email erikatatianaayala@ufps.edu.co Vinculo en perfil de google scholar https://scholar.google.es/citations?user=-JAelLwAAAAJ\&hl=es

${ }^{3}$ Doctorado en curso - Docente TC Dpto. de Arquitectura Diseño y Urbanismo UFPS - Colombia. Investigadora Grupo de Investigación Taller de Arquitectura y Gestión del Territorio TAR_GET Categoría B Colciencias, Investigadora Junior Colciencias ORCID https://orcid.org/0000-0003-0331-0568 Email luzkarimecr@ufps.edu.co
} 
venido desarrollando estos procesos en mira a la Acreditación de alta calidad, ya que se considera de vital importancia generar estudios que soporten la pertinencia de los contenidos y las temáticas que se desarrollan en el interior de los programas académicos.

Dentro de este proceso, se establecen acciones encaminadas a la autoevaluación del currículo que soporta el Proyecto Educativo del Programa (PEP). Esto teniendo en cuenta que el currículo es el instrumento que contiene la estructura del conocimiento y las competencias que el estudiante debe adquirir durante su educación de pregrado. En el currículo se condensan los objetivos, las habilidades y las actitudes que debe adquirir el estudiante para su desempeño académico y de ejercicio profesional, así como las diversas metodologías utilizadas al interior de los programas para la formación profesional de los estudiantes en pro de la construcción del conocimiento (Molina Natera, 2012; Tami Cortes y Coronel Ruiz, 2015).

Así las cosas, es importante reseñar que:

La palabra currículo proviene del latín currículum, de currere, que significa "carrera". El concepto de currículo ha variado a través del tiempo, acompañando las transformaciones sociales, históricas, científicas y tecnológicas de la escuela, así como su campo disciplinar ha tenido cambios en sus estructuras y estrategias según los objetivos de la educación. (ACFA, 2016, p.17)

Asimismo, dentro de la Ley General de Educación se establece que los currículos corresponden al conjunto de criterios, programas y metodologías encaminados a la formación integral y la construcción de la identidad local, regional y nacional, mediante el cumplimiento de los ejes misionales de una institución, direccionada a la formación integral de los seres humanos bajo un perfil profesional específico (ACFA, 2016). Por lo tanto, el proceso de autoevaluación, revisión y ajuste de los currículos es una tarea permanente que debe ser realizada por parte de las unidades académicas, con el fin de que los currículos se encuentren actualizados y respondan a los cambios, los requerimientos del contexto y a la tecnología que hace parte de la sociedad (Pontificia Universidad Católica de Valparaiso, 2015).

Desde la disciplina de la Arquitectura, los currículos contemplan competencias genéricas de formación fundamental, competencias específicas disciplinares y competencias específicas profesionales; por medio de las cuales se formulan los perfiles de egreso de los futuros arquitectos; lo anterior a través de ambientes de aprendizaje por medio de los cuales se puedan llegar a adquirir competencias comunicativas, de capacidad crítica- argumentativa, de trabajo en equipo y habilidades para el desempeño creativo en diversos entornos multiculturales (Urbina Cardenas, 2010). De esta manera, los programas de arquitectura deben presentar una relación coherente entre los propósitos de formación, las competencias generales y específicas y las áreas del currículo, con respecto a las sub áreas estipuladas como diseño arquitectónico, urbanismo, técnica, historia, expresión gráfica y sociohumanística, por medio de las cuales se estructura el currículo del programa (Universidad Francisco de Paula Santander, 2018). Bajo este orden de ideas, se debe recordar que dentro de la arquitectura es importante comprender la relación del ser humano con el espacio urbano, este último entendido como el lugar en el que se desarrolla la vida urbana, en el cual se llevan a cabo actividades económicas, políticas, sociales y culturales propias de los asentamientos humanos densificados, por medio de la utilización de infraestructura y una red de servicios que permiten la convivencia de las personas (Ayala Garcia, 2017) hecho por el cual se presenta como uno de los aspectos de estudio más relevante dentro de la formación de los estudiantes de arquitectura. En este sentido, el objetivo del presente artículo consiste en reconocer las bases conceptuales que sustentan el currículo del programa de arquitectura de la UFPS con respecto a la noción de espacio urbano. 


\section{Metodología}

La metodología de este artículo corresponde a un enfoque cualitativo, entendido como un proceso centrado en la recolección y el análisis de los datos mediante una acción indagatoria basada en los hechos y su interpretación, a través del cual se produce un proceso lógico, inductivo en el que se explora, se describe y finalmente se generan perspectivas teóricas (Hernández Sampieri, 2014). Su fuente de obtención de datos es documental, fue elaborada bajo una dimensión de profundidad (Peña Vera y Pirela Morillo, 2007) centrada en el analisis de los aspectos, elementos y características más relevantes del currículo del programa de Arquitectura de la UFPS (syllabus) relacionados con el espacio urbano como categoría de estudio. Finalmente, la metodología según su diseño corresponde a un método interpretativo, basado en una inducción analítica.

El marco metodológico empleado para llevar a cabo esta investigación, comprendió el análisis de la malla curricular del programa de Arquitectura de la UFPS, la identificación del ciclo formativo del currículo, el establecimiento de las líneas formativas que dan origen a la triada teórica (Historia VI, Taller VI y Diseño Urbano) y el semestre académico (Semestre VI) en donde se presenta mayor relación con la categoría de estudio propia de esta investigación; posteriormente se realiza la comparación del syllabus de la triada teorica con respecto a los componentes generales de evaluación del ICFES, que permiten establecer categorías de análisis que responden al concepto de espacialidad urbana.

La investigación contó con un soporte documental, las fuentes de información obedecieron a la búsqueda de fuentes primarias y autores relevantes; por su parte, también se utilizarón fuentes secundarias como libros, tesis, artículos e informes de renovación de registro y Acreditación en Alta Calidad; mediante la utilización de bases de datos bibliográficos y revistas indexadas. La base conceptual que oriento este estudio corresponden a: Arquitectura, Currículo, Educación y Espacio urbano.

En el plan de redacción del presente artículo, los resultados se estructuran a partir de 3 títulos. El primero denominado "Espacio urbano y ciudad" en el cual se aborda la noción de espacio urbano y su relevancia dentro de la organización de la ciudad, consecuentemente se explica la importancia de su estudio dentro del currículo de arquitectura y se aborda desde tres enfoques: el espacio urbano como escenario, el espacio urbano como entidad y el espacio urbano y la educación. El segundo titulo denominado "El espacio urbano desde el curriculo del programa de Arquitectura de la UFPS" establece una línea de razonamiento a partir de categorías que permiten el reconocimiento de las asignaturas en las cuales se presentan la percepción, comprensión y el reconocimiento del espacio urbano en el programa de Arquitectura de la UFPS; y el tercer título denominado "La triada teórica a través de los componentes generales de evaluación del Instituto colombiano para la evaluación de la educación -ICFES" en donde se determinan categorías de análisis para las asignaturas que componen la triada teórica por medio de los componentes generales de evaluación del ICFES, realizando un análisis a profundidad de la relación del concepto de espacio urbano y el currículo de arquitectura de la UFPS.

\section{Resultados}

\subsection{El espacio urbano y la ciudad}

La comprensión de la ciudad corresponde en sí misma al entendimiento del espacio urbano. Esto al tener en cuenta que el espacio urbano es un lugar de habitación, que conjuga lo construido y lo no construido, es un lugar político que configura lo público y permite el encuentro ciudadano (Fundación Rogelio Salmona, 2014). El espacio urbano puede ser abordado desde una mirada política, ecológica y psicológica, a través de la cual se puede definir como un espacio relacional, construido y organizado por los individuos, mediante procesos centrados en la experiencia y relacionados con el entorno que lo rodea. De esta manera, las diferentes formas de abordar el 
espacio urbano permiten configurar un adecuado ordenamiento urbano en equilibrio a las funciones de la ciudad (Cordova Bojorquez y Romo Aguilar, 2015).

En este sentido, los arquitectos como actores sociales deben desde su formación de pregrado reconocer la importancia del espacio urbano. Por tal razón, los currículos que acompañan la formación académica de los futuros arquitectos deben considerar lo urbano como un aspecto fundamental presente en las competencias que acompañan el quehacer académico y profesional de la arquitectura, teorizado desde las subáreas de formación tales como el diseño arquitectónico, el urbanismo, la técnica, la historia, la expresión gráfica y la sociohumanística; por medio de las cuales se logra en los ciclos de formación básico, profundización y profesionalización una adecuada contextualización, una proyección y una materialización del espacio (Universidad Nacional de Colombia, 2013).

Desde esta perspectiva, la implementación de estrategías tendientes al análisis del espacio urbano pueden llegar a promover el entendimiento de la relación entre las estructuras culturales y los entornos aquitectónicos. Lo anterior al tener en cuenta que la arquitectura se construye dentro de una sociedad en la cual se desarrolla la experiencia del ser humano (Acuña, 2005). Desde el componente historia de la arquitectura, es importante reseñar que para la arquitectura romana, el espacio (físico-construido) poseía características morfológicas urbanas determinadas como calles, equipamientos, infraestructuras y edificios, a las cuales reconocían como "urbs", estableciendo así el espacio construido como una unidad politico-administrativa a la que los griegos denominaban "polis" (Capel Saez, 2003)

La estructura física y vida humana son dos aspectos que siempre han ido unidos a nuestra manera de entender la ciudad, pero la relación entre esas dos formas de entender y la prioridad entre los dos aspectos (...) varía con el tiempo y con la situación. Cuando los atenienses decían polis se referían primordialmente a "la comunidad humana" y sólo en segundo lugar a "la estructura o entorno físico". Los romanos por su parte usaban la palabra civitas casi exclusivamente en la acepción humana. (Ramirez, 1998)

Por su parte, la perspectiva Aristotélica define a la urbe como "la huella que en el espacio deja la ciudad, el entramado que sirve o ha servido de protección a la comunidad política, a sus espacios de interacción y encuentro colectivo" (Arenas, 2013). Entonces el diseño y trazado de la urbs corresponden con la concepción del mundo que una determinada formación social tiene en un tiempo y un espacio específico.

De esta manera, definir el espacio urbano se torna una tarea compleja al tener en cuenta que como concepto puede ser abordado desde múltiples perspectivas; por tal razón, dentro de la presente investigación la relación del espacio urbano con la educación en la arquitectura se establece a partir de las competencias básicas que debe poseer el arquitecto dentro de su proceso académico y profesional, con respecto a temáticas como el diseño arquitectónico, el urbanismo, la historia y la sociohumanística. Para dar cumplimiento a tal fin, el espacio urbano es abordado a partir de tres enfoques: como escenario de aprendizaje, como unidad y desde la educación.

El espacio urbano como escenario. El escenario al igual que el espacio urbano opera como un contenedor de movimientos o acciones ejecutadas por determinados usuarios o habitantes llamados personajes, Esta relación le confiere una connotación eminentemente diegética, según la terminología de Gerard Genette (1989), puesto que se presenta indispensable para el desarrollo de una historia y la concatenación de eventos narrativos. El espacio escénico hace referencia al espacio diegético. Por lo tanto, es el medio en el que la realidad aparece representada, mientras que el espacio como escenario no es otro que el espacio diegético en su vertiente sustancial (lo propiamente representado) es decir, que el lugar debe entenderse como espacio físico y construido (Gennette , 1989). Es así como el espacio urbano puede interpretarse como espacio diegético o escenario. Esto 
al tener en cuenta que el mismo está delimitado por atributos tangibles; físicos, construidos, y representados, y por consiguiente es poseedor de forma y estética. En ese orden de ideas, el espacio urbano puede ser percibido a través de los sentidos (Carrasco Rey, 2004).

El espacio urbano como entidad. Con el tiempo se ha empezado a configurar una línea de investigación en la que se le otorga al espacio urbano y a la ciudad una nueva concepción, entendiéndolos como una entidad propia y como un paisaje emocional, en otras palabras "un escenario capaz de dialogar con un estado de ánimo" (Franco, 2017).

En este sentido, la imagen de la ciudad no se reduce a lo visible, a lo efectivamente construido, sino que abarca también el horizonte de referencia en el que se dan cita múltiples representaciones y relatos que construyen y reconstruyen, escriben y reescriben la ciudad y sus imaginarios. El espacio urbano actualiza y pone en escena un proyecto, un imaginario a través del cual se establecen las relaciones conceptuales mediante las cuales una sociedad, un colectivo o una comunidad piensa la ciudad y orienta la experiencia de ese espacio y de las relaciones entre quienes la habitan". (Lorente, 2012, p.596)

De esta forma puede el espacio urbano ser estudiado a partir de aspectos técnicos -proyectuales, constructivos, operativos- e histórico-geográficos propios de las infraestructuras en la ciudad, así como es posible analizar su compleja interrelación con el funcionamiento de la urbs. Es decir, los aspectos urbanísticos y sociales, las interrelaciones transporte-ciudad, bajo categorías de estudio como la vialidad, la accesibilidad, la movilidad y la conectividad, junto con otros conceptos derivados del saber urbanístico como la estructura urbana, la centralidad, densidad, forma, función, privacidad, adaptación, escala, espacio público, usos urbanos, entre otros (Santos y Ganges y De las Rivas , 2008). De esta manera, la ciudad se ha reconstruido como un artefacto narrativo y también como una estrategia discursiva destinada a indagar el sentido de las tensiones y conflictos que caracterizan los procesos de transformación del espacio urbano (Lorente, 2012).

El espacio urbano y la educación. El espacio urbano se presenta como un escenario de aprendizaje en la educación, al tener en cuenta que el mismo favorece la enseñanza que acompaña la complejidad de la ciudad y a su vez promueve su entendimiento, mediante la comprensión de los fenómenos físico- ambientales, socialculturales y económico-normativos que la acompaña. La puesta en práctica de este proceso estructura relaciones entre la comunidad y el territorio, que permiten la transformación física y social de las ciudades, así como el mejoramiento de la calidad de la educación, al abordar temáticas relevantes del contexto en el que los estudiantes y la población se desenvuelven. Todo lo anterior se ve reflejado en la formulación del espacio a través de proyectos que dentro de sus criterios de diseño tomen consideraciones con respecto al entorno, generando así una respuesta asertiva a la realidad del territorio.

A partir del siglo XX en Colombia se tomaron como referencia de estudio y proyección modelos pedagógicos estado unidenses sin tener en cuenta que la mayoría de ellos no daban cumplimiento a la realidad del contexto colombiano; Sin embargo, a mediados del siglo XX se incorporó un nuevo modelo pedagógico basado en el auto aprendizaje de los estudiantes. En siglo XXI se implementó un modelo pedagógico enfocado en el desarrollo más no en el aprendizaje, logrando que los estudiantes asumieran un rol activo, mientras que el docente un rol mediador, logrando así que el conocimiento sea construido por el estudiante (Carrasquilla Villaraga, 2018).

Con relación a los modelos pedagógicos se considera que "El proceso de enseñanza-aprendizaje de acuerdo con los sistemas educativos vigentes en cada momento de su historia, genera respuestas diversas en el campo arquitectónico" (Maldonado Tapias, 1999, p.171). Las infraestructuras que ocupaban estos modelos no fueron actualizadas tal como las mismas pedagogías lo hacían, las aulas no eran más que simple espacios para impartir una clase y el patio para el recreo. (Carrasquilla Villaraga, 2018, p.22) 


\subsection{El espacio urbano desde el currículo del programa de Arquitectura de la UFPS}

Dentro del presente apartado se realiza un análisis documental a partir del syllabus del programa de Arquitectura de la UFPS, a través del cual se identifican las asignaturas que presenta un mayor énfasis en la percepción, la compresión y el reconocimiento del espacio urbano. El currículo del programa de Arquitectura de la UFPS se encuentra conformado por diez semestres de formación académica, cuenta con 143 créditos obligatorios y 20 créditos electivos, y se estructura a partir de tres ciclos de formación: El ciclo básico que comprende los semestres I, II y III (51 créditos); el ciclo de profesionalización correspondiente a los semestres IV, V y VI (48 créditos), y por último el ciclo de profundización con los semestres VII, VIII, IX y X (64 créditos) (Universidad Francisco de Paula Santander, 2018). Como se puede apreciar en el Cuadro 1, en el ciclo formativo básico con respecto a la temática de espacio urbano, se presentan materias con baja y mediana relevancia como: matemáticas, técnicas de diagramación y técnicas de expresión a mano alzada, Historia del Arte y la Arquitectura Antigua y Taller I Configuración Espacial, así como materias institucionales- obligatorias.

\section{Cuadro 1}

Currículo del Programa de Arquitectura de la UFPS.

Estructura Formativa ciclo básico.

\begin{tabular}{|c|c|c|c|}
\hline \multicolumn{3}{|c|}{ PRIMER SEMESTRE } \\
\hline Código & Nombre & Horas & Cred. \\
\hline 1500110 & HISTORIA DEL ARTE YLAARQUITECTURA ANTIGUA & 4 & 3 \\
\hline 1500111 & MATEMÁTICAS: GEOMETRIAY ARQUITECTURA & 2 & 2 \\
\hline 1500112 & TÉCNICAS DE DIAGRAMACIONY PRESENTACION & 2 & 2 \\
\hline 1500113 & TECNICAS DE EXPRESIÓNYMANO ALZADA & 2 & 2 \\
\hline 1500114 & TALLER I CONFIGURACIÓN ESPACIAL & 8 & 5 \\
1500115 & TÉCNICAS DE ESTUDIO & 2 & 2 \\
\hline 1500116 & INTRODUCCIÓN A LA VIDA UNIVERSITARIA & 1 & 1 \\
\hline
\end{tabular}

\begin{tabular}{|c|c|c|c|}
\hline \multicolumn{3}{|c|}{ SEGUNDO SEMESTRE } \\
\hline Código & Nombre & Horas & Cred. \\
\hline 1500210 & HISTORIA DEL ARTEYLAARQUITECTURA MEDIEVAL & 4 & 3 \\
\hline 1500211 & GEOMETRÍA DESCRIPTIVA & 2 & 2 \\
\hline 1500212 & FOTOGRAFÍA DIGITAL & 2 & 2 \\
\hline 1500213 & EXPRESIÓN DOS DIMENSIONES & 2 & 2 \\
\hline 1500214 & TALLER II ESPACIO PENETRABLE & 8 & 5 \\
\hline 1500215 & ESTRATEGIASYPROCESOS DE DISEÑO & 2 & 2 \\
\hline 1500216 & ESTRUCTURASI & 2 & 2 \\
\hline
\end{tabular}

\begin{tabular}{|c|c|c|c|}
\hline \multicolumn{3}{|c|}{ TERCER SEMESTRE } \\
\hline Código & Nombre & Horas & Cred. \\
1500310 & HISTORIA DEL ARTEY LA ARQUITECTURA PRINCIPIOS & 4 & 3 \\
\hline 1500311 & DE LAMODERNIDAD & 2 & 2 \\
\hline 1500312 & TALLER III PEQUEÑO FORMATO & 8 & 5 \\
\hline 1500313 & ESTRUCTURASII & 2 & 2 \\
\hline 1500314 & SOCIOLOGÍAI & 2 & 2 \\
\hline 1500315 & CONSTRUCCIÓN DEL CONOCIMIENTO & 2 & 2 \\
\hline
\end{tabular}

\begin{tabular}{|c|}
\hline RELEVAMCIA MATRRIAS \\
\hline ALTA \\
\hline MEDLANA \\
\hline BANA \\
\hline INSTINUCIONAL OBLIOATORLA \\
\hline
\end{tabular}

Fuente: Elaboración propia a partir de Universidad Francisco de Paula Santander (2018). 
En el cuadro 2 se puede observar que en el ciclo formativo de profesionalización propio de los semestres IV, V y $\mathrm{VI}$, se ofrece una mayor proporción de materias de alta relevancia con relación a la temática de espacio urbano y menos materias de mediana y baja relevancia (en este ciclo solo se ofrece una materia institucionalobligatoria). Además de esto, se espera que en este ciclo exista una mayor comprensión teórico-práctica por parte del estudiantado con respecto al espacio urbano.

\section{Cuadro 2}

Currículo del Programa de Arquitectura de la UFPS Estructura Formativa ciclo profesionalización

\begin{tabular}{|c|c|c|c|}
\hline \multicolumn{3}{|c|}{ CUARTO SEMESTRE } \\
\hline Código & Nombre & Horas & Cred. \\
\hline 1500410 & HISTORIA DEL ARTEY LA ARQUITECTURAMODERNA & 4 & 3 \\
\hline 1500411 & DIBUJO ASISTIDO POR COMPUTADORESI & 2 & 2 \\
\hline 1500412 & TALLER IV REFLEXIONARYCOTIDIANIDAD & 8 & 5 \\
\hline 1500413 & CONCEPTOS BÁSICOS DE URBANISMO & 2 & 2 \\
\hline 1500414 & OBRA NEGRA & 2 & 2 \\
\hline 1500415 & INTRODUCCIÓNAL METODO CIENTIIFICO & 2 & 2 \\
\hline
\end{tabular}

\begin{tabular}{|c|c|c|c|}
\hline \multicolumn{3}{|c|}{ QUINTO SEMESTRE } \\
\hline Código & Nombre & Horas & Cred. \\
\hline 1500510 & HISTORIA DEL ARTEYLA ARQUITECTURA & 4 & 3 \\
\hline 1500511 & CONTEMPORÁNEA & 2 & 2 \\
\hline 1500512 & TALLER V REFLEXIONOR POMPUTADORESII & 2 & 5 \\
\hline 1500513 & URBANAS & 8 & 2 \\
\hline 1500514 & FORMACIÓNYEXTENSIÓN DE CIUDADES & 2 & 2 \\
\hline 1500515 & OBRA GRIS & 2 & 2 \\
\hline
\end{tabular}

\begin{tabular}{|c|c|c|c|}
\hline \multicolumn{3}{|c|}{ SEXTO SEMESTRE } \\
\hline Código & Nombre & Horas & Cred. \\
\hline 1500610 & $\begin{array}{c}\text { HISTORIA DEL ARTEYLA ARQUITECTURA } \\
\text { LATINOAMERICANA COLOMBIANA }\end{array}$ & 4 & 3 \\
\hline 1500611 & DIBUJO ASISTIDO POR COMPUTADORES III & 2 & 2 \\
\hline 1500612 & TALLER VIINTERSECCIONES URBANAS & 8 & 5 \\
\hline 1500613 & DISEÑO URBANO & 2 & 2 \\
\hline 1500614 & OBRA BLANCA & 2 & 2 \\
\hline 1500615 & SOCIOLOGIA III & 2 & 2 \\
\hline
\end{tabular}

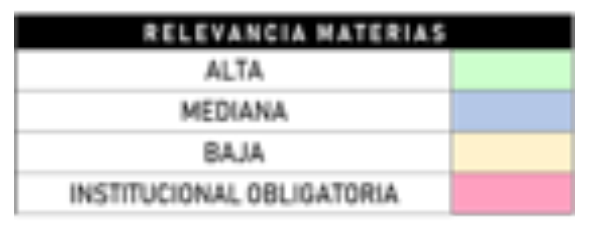

Fuente: Elaboración propia a partir de Universidad Francisco de Paula Santander (2018).

A partir del ciclo de profundización la experiencia educativa del estudiante cambia. El proceso establecido por el programa, le lleva a elegir una rama específica de la Arquitectura en la que le interese continuar su formación. Como se puede apreciar en el Cuadro 3, se presenta una nueva modalidad de materia denominada "Taller Vertical"; que es en esencia una continuación del formato "Taller de Diseño" (ciclo básico y ciclo de profesionalización), adaptado a un tema de profundización particular. Esta diversificación en la malla curricular, 
así como la aparición de materias Electivas con enfoques propios, permite establecer que durante los semestres VII, VIII, IX y X el estudio de espacio urbano se presenta con una relevancia medio-bajo, mediante el esquema (1:2) Es decir, una asignatura de relevancia mediana y dos de relevancia baja (relacionados con el espacio urbano mediante análisis documental del syllabus) por semestre academico.

Cuadro 3

Currículo del Programa de Arquitectura de la UFPS

Estructura Formativa ciclo profundización.

\begin{tabular}{|c|c|c|c|}
\hline \multicolumn{3}{|c|}{ SEPTIMO SEMESTRE } \\
\hline Código & Nombre & Horas & Cred. \\
\hline 1500710 & TALLER VERTICAL I & 8 & 5 \\
\hline 1500711 & ELECTIVA PROFUNDIZACIÓN I & 4 & 3 \\
\hline 1500712 & DISEÑO AMBIENTAL & 2 & 2 \\
\hline 1500713 & INSTALACIONESTÉCNICAS & 2 & 2 \\
\hline 1500714 & ELECTIVA IFLEXIBILIZACIÓN & 2 & 2 \\
\hline 1500715 & INGLÉSI & 2 & 2 \\
\hline
\end{tabular}

\begin{tabular}{|c|c|c|c|}
\hline \multicolumn{3}{|c|}{ OCTAVO SEMESTRE } \\
\hline Código & Nombre & Horas & Cred. \\
\hline 1500810 & TALLER VERTICAL II & 8 & 5 \\
\hline 1500811 & ELECTIVA PROFUNDIZACIÓN II & 4 & 3 \\
\hline 1500812 & PRESUPUESTOY PROGRAMACIÓN & 2 & 2 \\
\hline 1500814 & INGLÉS II & 2 & 2 \\
\hline 1500815 & CONSTITUCIÓNYCIVISMO & 2 & 2 \\
\hline 1500816 & ELECTIVA II FLEXIBILIZACIÓN & 2 & 2 \\
\hline
\end{tabular}

\begin{tabular}{|c|c|c|c|}
\hline \multicolumn{3}{|c|}{ NOVENO SEMESTRE } \\
\hline Código & Nombre & Horas & Cred. \\
\hline 1500910 & TALLER VERTICAL III & 8 & 5 \\
\hline 1500911 & ELECTIVA PROFUNDIZACIÓN III & 4 & 3 \\
\hline 1500912 & GESTIÓN I & 2 & 2 \\
\hline 1500914 & INGLÉS III & 2 & 2 \\
\hline 1500915 & METODOLOGIA DE LA INVESTIGACIÓN & 2 & 2 \\
\hline 1500916 & ELECTIVA III FLEXIBILIZACIÓN & 2 & 2 \\
\hline
\end{tabular}

\begin{tabular}{|c|c|c|c|}
\hline \multicolumn{3}{|c|}{ DECIMO SEMESTRE } \\
\hline Código & Nombre & Horas & Cred. \\
\hline 1501010 & TALLER VERTICALIV & 8 & 5 \\
1501011 & ELECTIVA PROFUNDIZACIÓN IV & 4 & 3 \\
\hline 1501012 & GESTIÓN II & 2 & 2 \\
\hline 1501014 & ETICA PROFESIONAL & 2 & 2 \\
\hline 1501015 & SEMINARIO DE INVESTIGACIÓN & 2 & 2 \\
\hline 1501016 & PROYECTODE GRADO & 10 & 0 \\
\hline 1501017 & ELECTIVA IVFLEXIBILIZACIÓN & 2 & 2 \\
\hline 1501081 & IMAGENESYREPRESENTACIONES GRÁFICAS & 4 & 3 \\
\hline
\end{tabular}

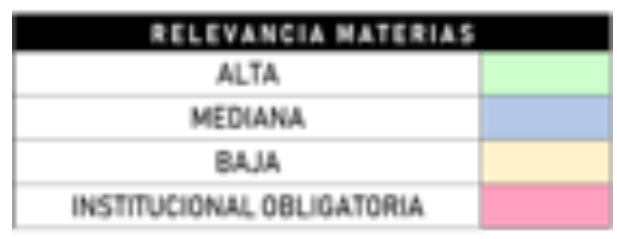

Fuente: Elaboración propia a partir de Universidad Francisco de Paula Santander (2018). 
Según el número de materias, se resalta que en el $X$ semestre el estudiante del programa de Arquitectura de la UFPS puede optar por cursar siete materias más el proyecto de grado; mientras que en los semestre I, II y IX puede cursar siete materias, y en los restantes seis materias. Con respecto a la intensidad horaria, el currículo de Arquitectura presenta sus índices más altos en el VI y X semestre con veinticuatro horas, seguido del semestre II con veintidós horas, primer semestre con veintiuna horas y los restantes con una intensidad de veinte horas, respectivamente. Finalmente, con respecto a los créditos se establece que su mayor representación corresponde al semestre II con dieciocho créditos, seguido del semestre I con diecisiete créditos, semestres III, IV, V, VI, VII y VIII con dieciséis créditos y semestre $X$ con catorce créditos. Esta etapa de reconocimiento y análisis del currículo del programa de Arquitectura de la UFPS, actúa como Primer Filtro dentro del proceso de identificación de categorías, y permite identificar la siguiente tendencia.

Cuadro 4

Currículo del Programa de Arquitectura de la UFPS

Selección ciclo profesionalización.

\begin{tabular}{|c|c|c|c|c|c|c|c|c|c|c|}
\cline { 2 - 12 } \multicolumn{1}{c|}{} & \multicolumn{4}{c|}{ BÁSICO } & \multicolumn{3}{c|}{ PROFESIONALIZACIÓN } & \multicolumn{4}{c|}{ PROFUNDIZACIÓN } \\
\hline SEM & $\mathrm{I}$ & $\mathrm{I}$ & $\mathrm{II}$ & $\mathrm{IV}$ & $\mathrm{V}$ & $\mathrm{VI}$ & $\mathrm{VII}$ & $\mathrm{VIII}$ & $\mathrm{IX}$ & $\mathrm{X}$ \\
\hline MATERIAS & 7 & 7 & 6 & 6 & 6 & $\mathbf{6}$ & 6 & 6 & 6 & $7^{*}$ \\
\hline HORAS & 21 & 22 & 20 & 20 & 20 & $\mathbf{2 4}$ & 20 & 20 & 20 & 24 \\
\hline CRÉDITOS & 17 & 18 & 16 & 16 & 16 & $\mathbf{1 8}$ & 16 & 16 & 16 & 14 \\
\hline
\end{tabular}

En el semestre $X$ el estudiante puede cursar 7 materias más proyecto de grado $\left(7^{*}\right)$

Fuente: Elaboración propia a partir de Universidad Francisco de Paula Santander (2018).

De esta manera, se establece que dentro del ciclo de profesionalización se presentan con mayor relevancia las materias relacionadas con la temática del espacio urbano; Por lo tanto para efectos de la presente investigación se excluyen los ciclos básico y de profundización. Esto, teniendo en cuenta que la relevancia temática de estos dos ciclos corresponde a otras competencias en la educación del Arquitecto que no serán tenidas en cuenta en el desarrollo de este artículo.

La segunda estrategia o filtro que se establece en el proceso de análisis del currículo del programa de Arquitectura de la UFPS, identifica lo que en adelante será denominado como Líneas Formativas, entendidas como el conjunto de asignaturas que por su continuidad y recurrencia configuran ejes indispensables en la formación del estudiante de Arquitectura. A través de estas líneas formativas se identificarán posteriormente las categorías de análisis definitivas con respecto al espacio urbano.

Corresponden al ciclo básico las líneas formativas: Historia del Arte y la Arquitectura Antigua, Taller I configuración Espacial, Historia del Arte y la Arquitectura Medieval, Taller II Espacio Penetrable, Historia del Arte y la Arquitectura. Principios de la Modernidad y Taller III Pequeño Formato. Hace parte del ciclo de profesionalización las siguientes líneas formativas: Historia del Arte y la Arquitectura Moderna, Taller IV Reflexionar y Cotidianidad, Conceptos Básicos de Urbanismo, Historia del Arte y la Arquitectura Contemporánea, Taller V Reflexiones y Transformaciones Urbanas, Formación y Extensión de Ciudades, Historia del Arte y la Arquitectura Latinoaméricana y Colombiana, Taller VI Intersecciones Urbanas y Diseño Urbano. ${ }^{3}$

\footnotetext{
3 Teniendo en cuenta la diversificación del currículo de Arquitectura de la UFPS a razón de la introducción de los Talleres verticales y las electivas en esta parte del proceso de análisis del currículo no se tendrá en cuenta el ciclo de profundización.
} 


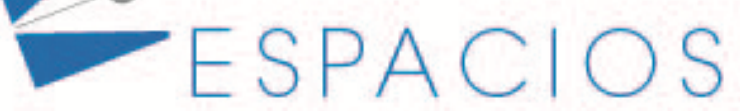

En ese orden de ideas, con respecto a la figura 1 es posible afirmar que el currículo del programa de Arquitectura de la UFPS, está articulado por (3) tres Líneas Formativas fundamentales: Historia, Taller de Diseño y Urbanismo, Estas Líneas Formativas fundamentales funcionan como ejes principales del PEP y alrededor de estas se relacionan e interconectan el resto de las materias del currículo, fortaleciendo los contenidos complementarios que corresponden a la formación integral del estudiante de Arquitectura.

Figura 1

Líneas Formativas Fundamentales del

Currículo de Arquitectura de la UFPS

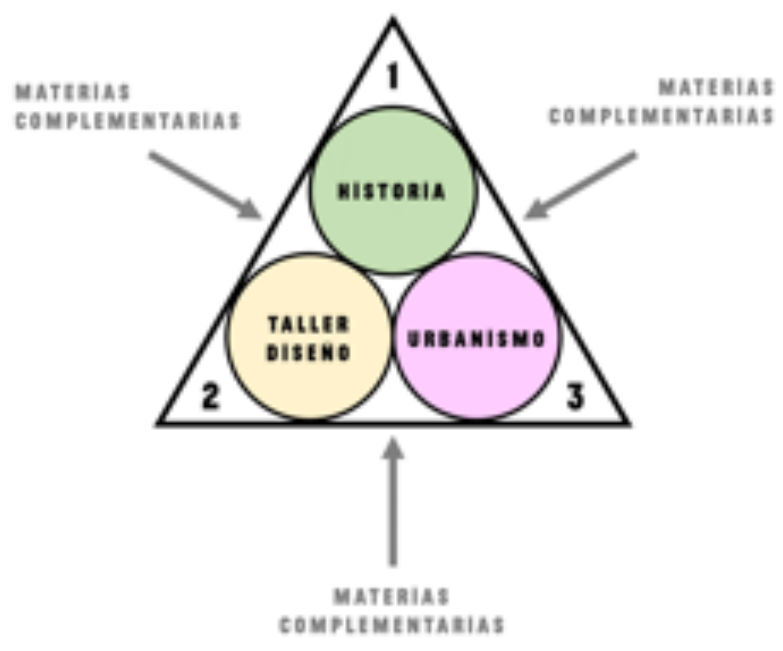

E JES PRIN CIPALES

Fuente: Elaboración propia

El siguiente paso para la identificación de categorías de análisis, reconoce los resultados encontrados en el primer y segundo filtro, según sus criterios respectivos, permitiendo definir las siguientes premisas:

Premisa 1 - Ciclo formativo con mayor relación al espacio urbano: ciclo de Profesionalización.

Premisa 2 - Líneas formativas fundamentales con mayor relación al espacio urbano: Historia / Taller de diseño / Urbanismo

Premisa 3 - Semestre con mayor relación al espacio urbano: Sexto semestre.

El sexto semestre diseñado por el programa de Arquitectura UFPS, no solamente es el de mayor intensidad horaria y mayor concentración de créditos en relación al número de materias, sino que se constituye como el semestre que da cierre al ciclo de profesionalización. Concluyen aquí también, las tres líneas formativas fundamentales identificadas como Ejes Principales del currículo. En este punto de la formación del Arquitecto se precisa que la teoría respecto del espacio urbano tome forma y se complemente a través del taller de diseño. Se resalta que los procesos pedagógicos de las líneas formativas convergen en este semestre, haciendo que funcione como un compendio de saberes.

El Fundamento Pedagógico dentro del Documento Maestro del Registro Calificado, citado en el Proceso Pedagógico de Taller de Diseño VI: Taller Intersecciones Urbanas, estipula que el Plan de estudios de Arquitectura opera desde "un enfoque pedagógico, dialógico-crítico, que potencializa las conexiones con otras disciplinas, 
generando un currículo integral y flexible" (Mariño, et al., 2017). Por su parte, los conceptos de conectividad, integralidad y flexibilidad en el currículo son evidentes al analizar con más detalle las tres líneas formativas fundamentales del sexto semestre, puesto que se reconocen en ellas perspectivas recurrentes, narrativas comparables y una profunda relación entre sus temáticas. Esta interacción entre materias será denominada para efectos de este artículo como "Tríada teórica", y a partir de su contexto se identificarán finalmente las categorías de análisis que responden al concepto de espacio urbano.

\subsection{La triada teórica a través de los componentes generales de evaluación del ICFES}

Los Componentes Generales de Evaluación establecidos por el ICFES “son los diferentes aspectos que siempre están presentes en todo proceso de proyectos (...) durante el proceso creativo corresponde a todo arquitecto conjugar los criterios propios a estos ejes con las características específicas de la situación" (Instituto Colombiano para la Evaluación de la Educación, 2019, p.9). Es de resaltar que estos componentes han sido implementados dentro de los procesos de formación y evaluación de las asignaturas del programa de Arquitectura de la UFPS, por tal razón para el presente artículo se configuran como un medio instrumental en la estructuración de las categorías de análisis definitivas, dado que operan como el epítome de un conjunto de criterios fácilmente diferenciables de los demás y aplicables en la percepción del espacio urbano. Al respecto, el ICFES bajo las competencias de conceptualizar, justificar y argumentar las define como:

Urbano-ambiental: Interpreta los espacios urbanos y arquitectónicos que atienden las necesidades habitacionales del ser humano. Valora los elementos naturales que hacen parte del contexto para involucrarlos en los proyectos arquitectónicos. Interpreta los elementos y sistemas urbanos como condiciones del diseño arquitectónico.

Formal-espacial: Integra el conocimiento de los modelos y la espacialidad que se genera para que aporten en la conceptualización de la propuesta.

Tecnológico: Coordina, analiza y sintetiza la información procedente de procesos técnicos involucrados en proyectos arquitectónicos. Analiza fenómenos físicos inherentes a la arquitectura para generar espacios habitables y confortables.

Funcional: Caracteriza y conceptualiza el programa con el cual se justifican las operaciones proyectuales de la arquitectura. Se espera que en el desarrollo de las ideas arquitectónicas el estudiante recurra a fundamentos teóricos, como herramientas de diseño, para intervenir el contexto físico, y exponga los criterios en que se basa la toma de cada decisión en los diferentes componentes para conformar el proyecto como un sistema complejo.

En este apartado se realiza un paralelo del syllabus propio de la triada teórica presente en el sexto semestre del currículo del programa de Arquitectura UFPS. De esta manera, se enlazan y determinan aspectos relevantes que se enmarcan dentro de las categorías globales del ICFES, y seguidamente se procede a generar un paralelo entre los objetivos generales y específicos de las asignaturas para finalmente, ahondar en los contenidos básicos o las unidades que las componen.

Como se puede apreciar en la Figura 2, dentro de este paralelo con respecto a la presentación del curso (contextualización), se puede observar que el factor que se presenta con mayor fuerza hace referencia al "funcional" entrelazando los aspectos propios de las asignaturas a las diferentes dinámicas propias del espacio urbano. Seguidamente, se destacan aspectos derivados de la configuración urbana o territorial asociada a la categoría "urbano- ambiental". Para finalmente resaltar aspectos derivados de las categorías "formal- espacial y tecnológica". 
Figura 2

Paralelo de contextualización de la triada teorica en el Semestre VI

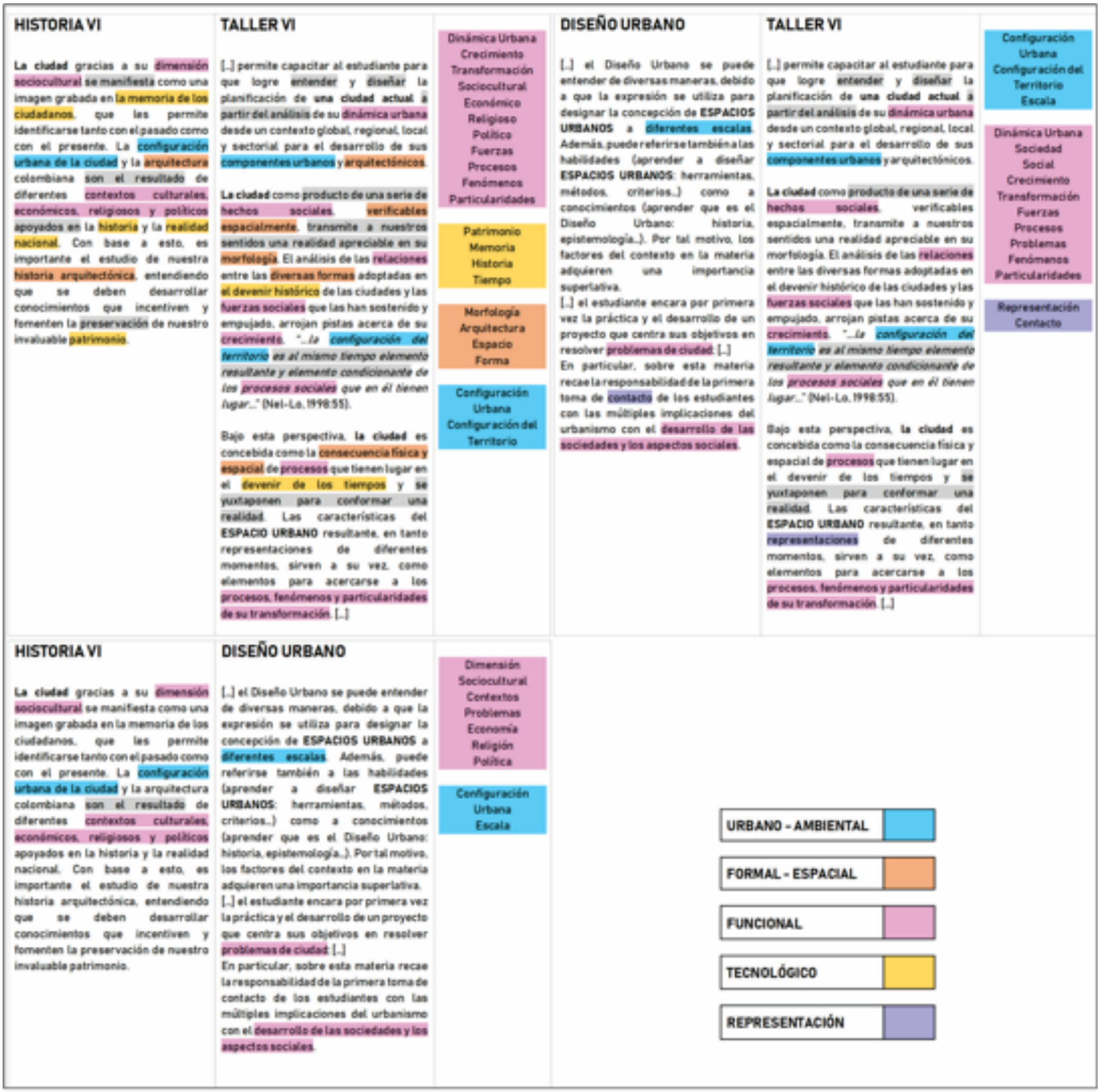

Fuente: Elaboración propia a partir del syllabus del programa de Arquitectura de la UFPS

Con respecto al análisis de los objetivos generales demarcados para estas asignaturas, se determina que con respecto al entendimiento del espacio urbano, las categorías con mayor incidencia corresponden a: "Funcional, urbano-ambiental y tecnológica". Para los objetivos específicos, se considera que las categorías de mayor impacto corresponden a la "urbano-ambiental y funcional". De la misma manera, el análisis de la triada teórica establece que para la comprensión del espacio urbano dentro de los contenidos básicos de las asignaturas estudiadas, se destaca en una mayor predominancia la categoría "funcional" a través de la identificación de variables como: Procesos de desarrollo, crecimiento, transformaciones de la ciudad, realidad urbana y dinámicas urbanas, estado actual de la ciudad, región, escenario. Finalmente, dentro de la categoría "formal espacial" se 
destacaron variables como: Lenguaje arquitectónico, determinantes de diseño, forma- morfología urbana y espacio público. En la categoria "tecnológica" se encuentran las técnicas de construcción y finalmente, en la categoría "urbano-ambiental" se observan: la composición urbanística, los trazados urbanos, los sistemas de parcelación y el componente medioambiental.

Los paralelos establecidos entre las materias de la tríada teórica según su contextualización, objetivos y sus contenidos básicos, exponen un razonamiento compartido sobre la comprensión del espacio urbano, recordando que la "urbs" es la ciudad tangible, ejemplificada por aspectos derivados de lo morfológico, lo físico, lo edificado y espacial. Por otro lado, dentro de este análisis se identifican los significados etimológicos referentes al binomio civitas + polis, que ejemplifica el aspecto intangible de la ciudad. Esto demuestra el interés del programa por construir un currículo integral que considera las dinámicas urbanas, los factores sociales, lo humano, lo sincrónico y lo diacrónico, "construyendo arquitectura desde y para el contexto" (Mariño, et al., 2017).

Consecuentemente, es pertienente resaltar la importancia del enfoque temporal con el que se lee, se percibe y se comprende la ciudad "urbs" (Espacio Urbano). Para tal fin, deben considerarse las siguientes afirmaciones extraídas de los paralelos en el ítem contextualización de las asignaturas Taller VI + Historia VI: Se dice de la ciudad, que es el "[...] producto de una serie de hechos sociales, verificables espacialmente." y que "[...] transmite a nuestros sentidos una realidad apreciable en su morfología". (Mariño, et al., 2017, p.3).

Figura 3

Espacio urbano tangible e intangible

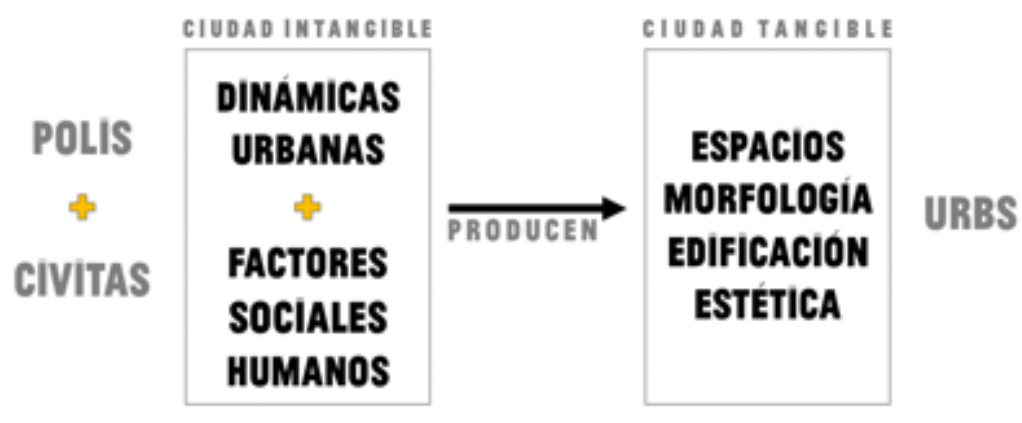

Fuente: Elaboración propia

Según la categorización establecida por la Universidad de Oxford esta lectura de la ciudad es de tipo sincrónico, dado que su enfoque reside en el producto manifestado (forma, espacio) y es verificable en el tiempo presente (dimensión tangible). El enfoque sincrónico en las categorías de análisis aborda fenómenos que se encuentra en un momento determinado, sin tener en cuenta su historia o su evolución en el tiempo (Universidad de Oxford, 2019). La comparación de los enfoques temporales sincrónico y diacrónico, subyacentes en las categorías de análisis, se presenta también en los contenidos básicos de la tríada teórica. Ambos son definidos en la unidad IV de la materia Diseño Urbano, en donde se les acuña el término "perspectiva".

Se establece que la perspectiva sincrónica es la forma actual que reconoce la diversidad de las áreas urbanas que la conforman, según sus características morfológicas, sus trazados, sistemas de parcelación, tipos arquitectónicos, etc. Asimismo, se define que la perspectiva diacrónica corresponde a la formación histórica, la permanencia, y las transformaciones que en ella ocurren o se derivan. Estableciendo así una lectura de la ciudad desde el enfoque diacrónico, el cual se ocupa de los hechos, fenómenos o circunstancias desde el punto de vista de su evolución en el tiempo (Universidad de Oxford, 2019). 
Así las cosas, esta evolución en el tiempo puede comprenderse a través de argumentos como: “[...] la configuración del territorio es al mismo tiempo elemento resultante y elemento condicionante de los procesos sociales que en él tienen lugar [...]" (Rodríguez, 1999, p.87), y "[...] la ciudad es concebida como la consecuencia física y espacial de procesos que tienen lugar en el devenir de los tiempos". (Mariño, et al., 2017, p.3).

Figura 4

Categorías de análisis

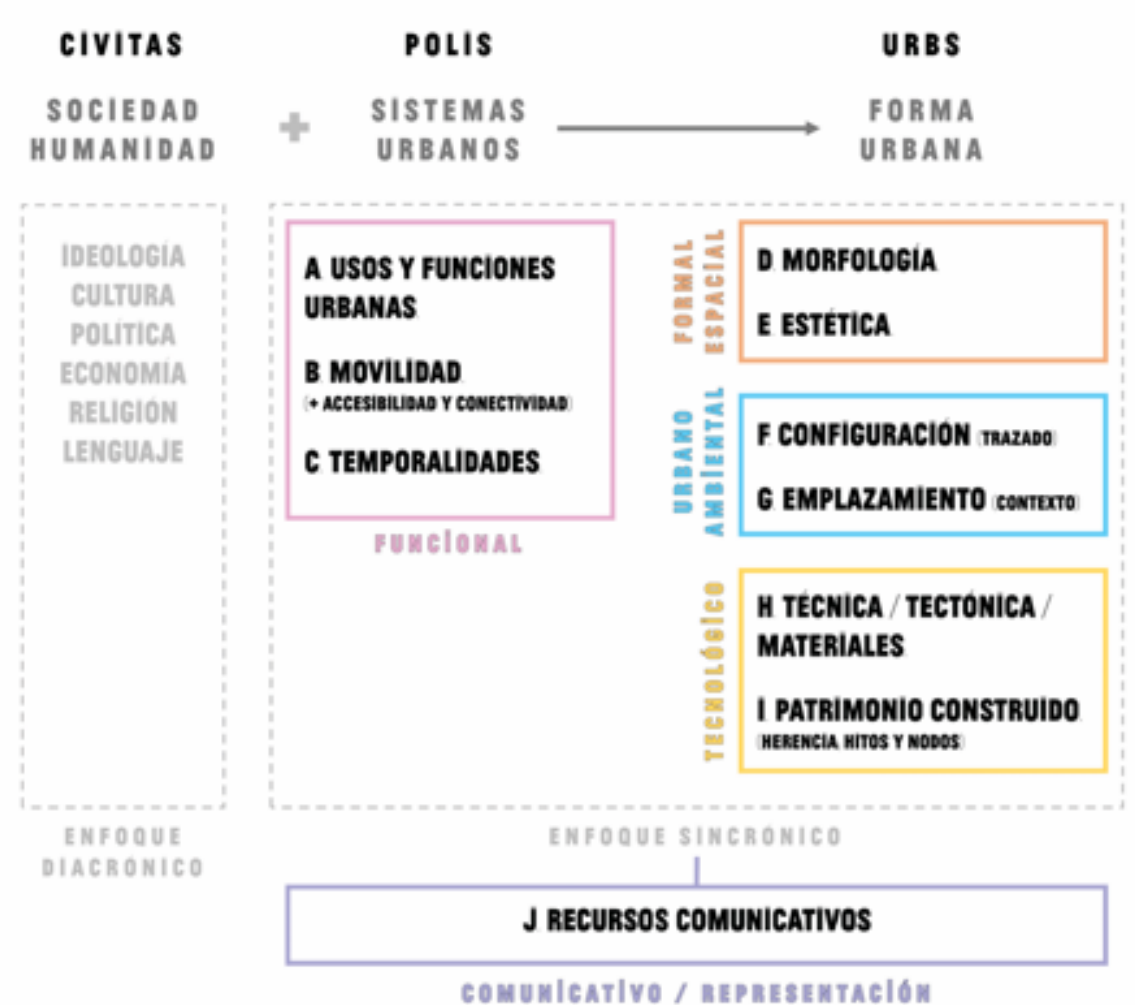

Fuente: Elaboración propia

Una vez realizado el análisis del currículo del programa de Arquitectura de la UFPS, a través del establecimiento de las tres (3) líneas formativas principales, así como de criterios de estudio tales como el significado etimológico de ciudad, la pertenencia a un componente general de evaluación reconocido por el ICFES, y la utilización de un enfoque temporal sincrónico; se establece que las categorías de análisis que responden al concepto de espacialidad urbana son: usos y funciones urbanas, movilidad, temporalidad, morfología, estética, configuración, emplazamiento, técnica, tectónica, materiales y patrimonio construido.

\section{Conclusiones}

En el presente artículo se analizó el currículo del Programa de Arquitectura de la UFPS, con el fin de identificar categorías de análisis relacionadas con la temática del Espacio Urbano. Para ello, se estableció una línea de razonamiento que permitió la organización de la información y la consecución de dicho objetivo. En la primera instancia del proceso, se encontró que el currículo del programa se divide en tres (3) ciclos de formación: Básico, Profesionalización y Profundización; respectivamente, distribuyendo así las materias y saberes de acuerdo a las competencias básicas que debe adquirir el estudiante de Arquitectura durante su formación. 
Se determinó que los ciclos de formación básico y de profundización no eran idóneos para la progresión de la identificación de categorías de análisis. En el ciclo básico, por ejemplo, se encontró una concentración muy baja de contenidos relevantes para el tema del espacio urbano. A pesar de que en este ciclo aparecen las primeras asignaturas de las líneas formativas fundamentales Historia del Arte y la Arquitectura y Taller de Diseño, los temas que se observan en el primero, segundo y tercer semestre de la carrera son de carácter introductorio y de muy baja complejidad urbana. Por otro lado, se identificó una alta especialización en los contenidos propuestos para el ciclo de profundización, apartándose del interés temático de esta investigación.

En ese sentido pudo reconocerse el ciclo de profesionalización como el más apto para la observación microcurricular extensiva, revelando en su estructura tres (3) líneas formativas o ejes principales subyacentes en todo el proceso de formación del Programa de Arquitectura de la UFPS: Historia del Arte y la Arquitectura, Taller de Diseño y Urbanismo (Triada Teorica). Se encontró que estas eran las líneas formativas con mayor concentración horaria, mayor recurrencia, mayor número de créditos y mayor relevancia temática en relación al espacio urbano. Estas líneas formativas crean una trayectoria que culmina en el sexto semestre del programa.

La observación de dicha trayectoria, enmarcada por el ciclo formativo de profesionalización, permitió identificar al sexto semestre como el más significativo del proceso, en relación con el interés investigativo de este proyecto. El sexto semestre funciona como el epítome o compendio de los saberes hasta ahora presentados y corresponde con un punto de la formación del arquitecto, en el cual se espera que el estudiante comprenda y reconozca conceptos más complejos e intersecciones sobre el espacio urbano.

Asimismo, fue posible observar la relación intrínseca y el funcionamiento integral de las tres líneas formativas, en lo que se denominó como la tríada teórica. Se encontró que las líneas de Historia VI, Taller de Diseño VI y Diseño Urbano, no solo presentan relevancia con la temática por sí solas, sino que se complementan y se corresponden entre sí, permitiendo generar múltiples paralelos y comparaciones que facilitaron la identificación de las categorías de análisis. Uno de los elementos fundamentales para el cruce de la tríada teórica fue el análisis del syllabus; un documento de gran utilidad que organiza los contenidos de cada materia según su contextualización, sus contenidos básicos, sus objetivos generales y específicos y otras acepciones de tipo operativo y estructural. Se halló que el diseño establecido a nivel institucional por la Universidad Francisco de Paula Santander resulta muy apropiado, conciso y pertinente para el desarrollo de investigaciones como la presente, puesto que se recopilan todos los datos instrumentales de las materias que desean estudiarse.

Por otro lado, el proceso de análisis del currículo permitió reconocer en el Programa de Arquitectura, un primer grupo de categorías que se fundamentan en las establecidas por el ICFES. Cada componente acuñado por el programa para la evaluación de las competencias del estudiante, corresponde con los lineamientos nacionales: Urbano-Ambiental, Formal-Espacial, Funcional y Tecnológico. Estas categorías globales a su vez, enmarcan subcategorías o categorías de análisis específicas que son evidentes por medio de los paralelos llevados a cabo dentro de la tríada teórica.

Finalmente se encontró, que las categorías de análisis específicas pueden agruparse en conjuntos de acuerdo con diversas variables: Según su relación con los significados etimológicos de la ciudad (Urbs, Civitas o Polis), según el enfoque temporal con que se observen (de tipo sincrónico o diacrónico), según su relación con el plano social, sistémico o formal del espacio urbano, y según su pertenencia a las categorías globales establecidas por el ICFES, presentes en el Programa de Arquitectura de la UFPS, cumpliendo de esta manera el proceso de autoevaluación que debe ser desarrollado por los programas académicos en pro de la calidad. 


\section{Referencias bibliográficas}

Acuña, P., 2005. Análisis formal del espacio urbano. Aspectos teóricos. Lima: Instituto de Investigación de la Facultad de Arquitectura y Urbanismo. Recuperado de: https://docplayer.es/5005221-Analisis-formal-delespacio-urbano-aspectos-teoricos-investigador-principal-percy-acuna-vigil.html

ACFA, 2016. Mallas curriculares de las Facultades de Arquitectura en Colombia. Documento de Trabajo. Bogotá: ACFA. Recuperado de: https://www.arquitecturaacfa.org/index.php/publicaciones/libros

Arenas, O. A., 2013. Urbe y ciudad: La necesaria distinción. Enfoques y perspectivas sociológicas. Antioquia: Universidad de Antioquia.

Ayala García, E. T., 2017. La ciudad como espacio habitado y fuente de socialización. Ánfora, 24(42), pp. 189216.

Capel Sáez, H., 2003. A modo de introducción: Los problemas de las ciudades. Urbs, civitas y polis. Mediterráneo económico, Volumen 3, pp. 9-22.

Carrasco Rey, R., 2004. Urbano, urbanidad y urbanismo. Urbanismos, Volumen 1, pp. 53-70.

Carrasquilla Villaraga, J. C., 2018. El Espacio Urbano Como Escenario de Aprendizaje Parque Educativo para la Formación "Casa Loma”, Bogotá: Universidad Católica de Colombia.

Cordova Bojorquez, G. y Romo Aguilar, M. d. L., 2015. Espacio urbano y actores sociales en la ciudad Chihuahua. México: Colegio de la Frontera Norte A.C.

Franco, A., 2017. Ciudad y espacio urbano en el último cine iberoaméricano. La Coruña: Universidad de Coruña.

Fundación Rogelio Salmona, 2014. Arquitectura y espacio urbano. Bogotá: Seminario de Arquitectura Latinoamericana SAL-15.

Gennette , G., 1989. Figuras III. Editorial Lumen.

Hernández Sampieri, R., 2014. Metodología de la investigación. 6 ed. México: Mc Graw Hill.

Instituto Colombiano para la Evaluación de la Educación, 2019. Guía de orientación: Saber pro competencias específicas. Módulo de proyecto de Arquitectura , Bogotá: ICFES.

Lorente, J. I., 2012. La reconstrucción fílmica de la urbe: Cine, memoria y ciudad. País Vasco, Universidad del País Vasco.

Maldonado Tapias, R., 1999. Historia de la arquitectura escolar en Colombia. Bogotá: Universidad Nacional de Colombia.

Mariño, J. A., Maldonado, A. E. y Díaz, Y., 2017. Syllabus Taller de diseño VI Intersecciones urbanas, Cúcuta: Programa de Arquitectura UFPS.

Molina Natera, V., 2012. Escritura a través del currículo en Colombia: Situación actual y desafíos. Magis Revista Internacional de Investigación en Educación, 5 (10).

Peña Vera, T. y Pirela Morillo, J., 2007. La complejidad del análisis documental. Información, cultura y sociedad: revista del Instituto de Investigaciones Bibliotecológicas, Issue 16, pp. 55-81.

Perilla Granados, J. S. A., 2018. Diseño curricular y transformación de contextos educativos desde experiencias concretas. Bogotá: Sergio Arboleda. Recuperado de: 
https://repository.usergioarboleda.edu.co/bitstream/handle/11232/1276/Diseño\%20curricular\%20y\%20tr ansformación.pdf?sequence=1\&isAllowed=y

Pontificia Universidad Católica de Valparaíso, 2015. Modelo curricular y lineamientos para el diseño curricular en pregrado. Valparaíso: Pontificia Universidad Católica de Valparaíso.

Ramírez, J. L., 1998. Los dos significados de la ciudad o la construcción de la ciudad como lógica y como retórica. Scripta Nova, 27 (1). Revista electrónica de Geografía y Ciencia Sociales. Recuperado de: http://www.ub.edu/geocrit/sn-27.htm

Universidad Francisco de Paula Santander, 2018. Informe de los estándares de calidad para la renovación del registro calificado Arquitectura Facultad de Educación, Artes y Humanidades Universidad Francisco de Paula Santander. Cúcuta: Universidad Francisco de Paula Santander.

Rodríguez, J., 1999. El palimpsesto de la ciudad: ciudad educadora. Armenia.

Santos y Ganges, L. y De las Rivas , J. L., 2008. Las ciudades con atributos: Conectividad, accesibilidad y movilidad. Ciudades, Issue 11, pp. 13-32.

Tami Cortes, M. D. y Coronel Ruiz, L. K., 2015. Análisis del impacto social del egresado del programa de arquitectura de la Universidad Francisco de Paula Santander entre los años 2010-2015. Revista Respuestas, 23(S1), pp. 76-80.

Universidad Nacional de Colombia, 2013. Proyecto Educativo de Programa autoevaluación y seguimiento de la calidad de los programas de pregrado Arquitectura, Manizales: Facultad de Ingeniería y Arquitectura. Recuperado de: http://www.fia.unal.edu.co/attachments/article/14/pep-arquitectura.pdf

Universidad Nacional de Colombia, 2014. Proceso de Autoevaluación, Bogotá: Dirección Nacional de Programas de Pregrado. Recuperado de: http://www.autoevaluacion.unal.edu.co/web/site/proceso

Universidad de Oxford, 2019. Lexico powered by Oxford. Recuperado de: https://www.lexico.com/?search_filter=en_dictionary

Urbina Cárdenas, J. E., 2010. El enfoque pedagógico de la UFPS: la perspectiva critica y dialógica como posibilidad para el mejoramiento de la docencia universitaria. Cúcuta: UFPS.

Esta obra está bajo una Licencia Creative Commons Attribución-NoCommercial 4.0 International

\section{(c) $)$ BY-NC}

